\title{
Muscle Conditional Medium Reduces Intramuscular Adipocyte Differentiation and Lipid Accumulation through Regulating Insulin Signaling
}

\author{
Haiyin Han, Wei Wei, Weiwei Chu, Kaiqing Liu, Ye Tian, Zaohang Jiang and Jie Chen * \\ College of Animal Science and Technology, Nanjing Agricultural University, Nanjing 210095, China; \\ 2014205001@njau.edu.cn (H.H.); wei-wei-4213@njau.edu.cn (W.W.); 2012205001@njau.edu.cn (W.C.); \\ 2015205001@njau.edu.cn (K.L.); 2016205005@njau.edu.cn (Y.T.); 2014105002@njau.edu.cn (Z.J.) \\ * Corresponding: jiechen@njau.edu.cn; Tel./Fax: +86-25-8439-9269
}

Received: 17 July 2017; Accepted: 14 August 2017; Published: 20 August 2017

\begin{abstract}
Due to the paracrine effects of skeletal muscle, the lipid metabolism of porcine intramuscular (i.m.) preadipocytes was different from that of subcutaneous (s.c.) preadipocytes. To investigate the development of i.m. preadipocytes in vivo, the s.c. preadipocytes were cultured with muscle conditional cultured medium (MCM) for approximating extracellular micro-environment of the i.m. preadipocytes. Insulin signaling plays a fundamental role in porcine adipocyte differentiation. The expression levels of insulin receptor (INSR) and insulin-like growth factor 1 receptor (IGF-1R) in i.m. Preadipocytes were higher than that in s.c. preadipocytes. The effects of MCM on adipocyte differentiation, lipid metabolism and insulin signaling transdution were verified. MCM induced the apoptosis of s.c. preadipocytes but not of s.c. adipocytes. Moreover, MCM inhibited adipocyte differentiation at pre-differentiation and early stages of differentiation, while the expression levels of INSR and IGF-1R were increased. Furthermore, MCM treatment increased adipocyte lipolysis and fatty acid oxidation through induction of genes involved in lipolysis, thermogenesis, and fatty acid oxidation in mitochondria. Consistent with the above, treatment of s.c. adipocytes with MCM upregulated mitochondrial biogenesis. Taken together, MCM can approximate the muscle micro-environment and reduce intramuscular adipocyte differentiation and lipid accumulation via regulating insulin signaling.
\end{abstract}

Keywords: intramuscular preadipocytes; extracellular micro-environment; insulin receptor; insulin-like growth factor 1 receptor; porcine; adipogenesis

\section{Introduction}

The deposition of fat in muscle, recognized by the intramuscular fat (IMF), is an important meat quality. Development of adipocytes located within muscles has been studied. A wide variety of evidence indicated significant differences in both metabolic and secretory functions between intramuscular adipocytes and subcutaneous adipocytes [1,2]. Consequently, intramuscular adipocytes had lower lipogenic enzyme activities, smaller cell sizes, and lower lipid content than subcutaneous adipocytes [3]. However, the mechanism underlying regional differences in adipogenesis still remains unknown.

The differentiation of preadipocytes to mature adipocytes includes activation of adipogenic gene expression and induction of insulin sensitivity [4]. Previous studies reported that insulin is an obligate hormone for preadipocyte differentiation [5-8]. Insulin signaling is initiated by binding of insulin to the insulin receptor (INSR), a receptor tyrosine kinase [9]. During adipocyte differentiation, the number of INSR increases approximately 25-fold [10]. Before the appearance of responsiveness of insulin receptor (INSR) to insulin during later stages of adipocyte differentiation, insulin mediates 
its early adipogenic effect via insulin-like growth factor-1 receptors (IGF-1R), which is initiated by activation of the tyrosine kinase at the cell surface [10]. Once triggered, the IGF-1R phosphorylates insulin receptor substrates-1 (IRS-1), a key cytosolic protein substrate, which subsequently activates downstream signal transduction pathways with impact on adipogenic gene transcription [11,12]. Meanwhile, insulin receptor was required for the full differentiation response because of the impaired adipocyte differentiation that was associated with decreased insulin receptor expression [13]. All of these findings suggested the molecular mechanism of insulin action on adipocyte differentiation, but the effects of insulin signaling on porcine intramuscular adipogenesis are not well understand. In this study, we turned our attention to the most proximal steps of insulin signaling for further study, including the INSR/IGF-1R and the major direct substrate, IRS-1.

Intramuscular adipocytes and subcutaneous adipocytes development occurs in the different extracellular micro-environment. Systemic blood circulation provides transport capabilities for endocrine hormones, cytokines (adipokines and myokines) and nutrition for adipose tissue development. However, the development of intramuscular adipocytes are exclusively affected by the paracrine activity of skeletal muscle fibers due to their particular location in close vicinity to muscle fibers. For example, myokines interleukin-15 (IL-15), myostatin (MSTN) and irisin inhibit adipocyte differentiation and fat deposition via a skeletal muscle to fat signaling pathway [14-16]. In these in vitro studies, it just verified the role of one purified cytokine present in the micro-environment. It is possible to approximate the tissue micro-environment using commercially available products. However, in all cases, it is unlikely that the material used represents the specific combination of factors that exist within the tissue micro-environment. To address these challenges, we extracted the interstitial fluid from skeletal muscle, which can be applied as muscle conditional cultured medium (MCM) for approximating the extracellular micro-environment of i.m. preadipocytes in vivo. The subcutaneous preadipocytes were cultured in the MCM to study intramuscular adipose tissue development, including adipocyte differentiation and adipose tissue expansion.

In the present study, the mechanism of insulin signaling in porcine adipocyte differentiation was verified. Meanwhile, the effects of extracellular micro-environment on adipocyte development were detected using skeletal muscle interstitial fluid that was applied as MCM. Furthermore, the molecular mechanism of MCM action on adipogenesis by modulating insulin signal transduction was investigated. The goal of this study was to understand the intramuscular adipogenesis with emphasis on cell micro-environment interactions that are pivotal in regulating adipocyte formation.

\section{Results}

\subsection{Cellular Differentiation and Identification of Porcine Intramuscular and Subcutaneous Preadipocytes}

The intramuscular (i.m.) and subcutaneous (s.c.) preadipocytes which isolated using the ceiling culture method were resuspended in culture medium with an irregular triangular appearance when freshly inoculated. After three days of culture in growth medium, both i.m. and s.c. preadipocytes showed fibroblast-like morphology (Figure 1A). Both of them could redifferentiate into mature adipocytes with lipid droplets following by adipogenic stimulation at confluence (Figure 1B). Pref- 1 is an adipocyte-specific protein, with a special expression in preadipocytes, inhibiting adipose differentiation in the early differentiation stage $[17,18]$. The fibroblast-like cells were identified by immunofluorescence staining of Pref-1. As shown in Figure 1C, positive Pref-1 reactions demonstrated that the isolated cells were preadipocytes with high purity. In conclusion, the date above confirmed that the fibroblast-like cells were porcine i.m. and s.c. preadipocytes, respectively. 


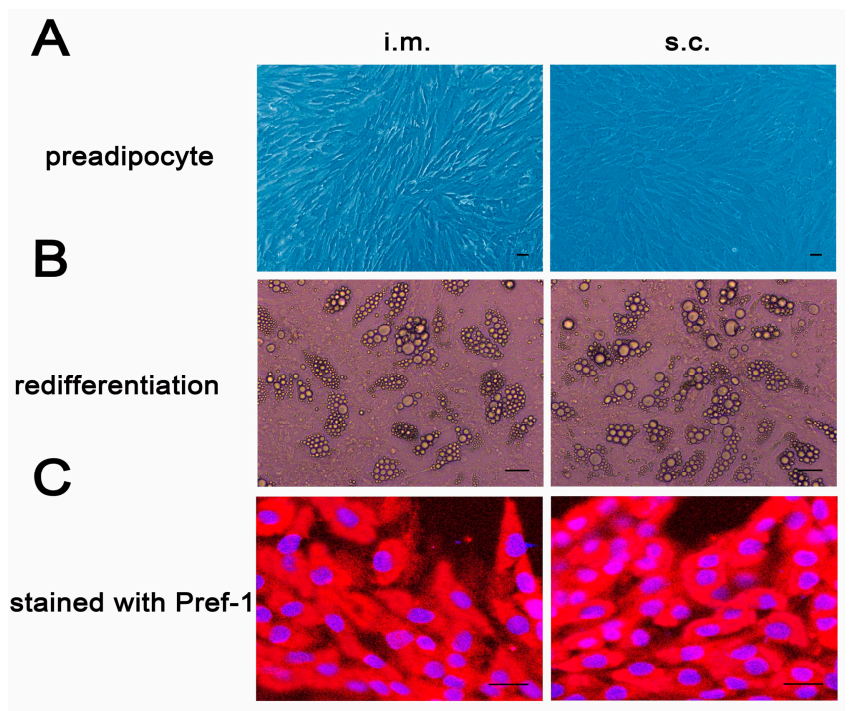

Figure 1. Identification of porcine i.m. and s.c. preadipocytes. The dedifferentiated porcine intramuscular (i.m.) and subcutaneous (s.c.) preadipocytes showed fibroblast-like morphology (A); The i.m. and s.c. preadipocytes were induced in adipogenic medium at confluence. Nine days later, the i.m. and s.c. preadipocytes were re-differentiated to mature adipocytes with lipid droplets (B); The isolated i.m. and s.c. preadipocytes were seeded in 12-well plates, and the cells of day 3 were detected by Pref-1 immunofluorescent staining (C). Scale bar $=100 \mu \mathrm{m}$.

\subsection{Inhibition Differentiation of Porcine Adipocyte by Downregulating the Expression of IGF-1R or INSR}

Insulin initiates its pleiotropic effects on cellular growth and metabolism by binding to its specific cell-surface receptor, INSR and IGF-1R. To elucidate the function of INSR and IGF-1R in determining adipocyte differentiation, s.c. preadipocytes were transfected with INSR and IGF-1R siRNAs, respectively. After 24 h, the s.c. preadipocytes were induced to differentiate following adipogenic stimulation.

Knockdown efficiency was examined by qPCR and immunoblotting for INSR and IGF-1R. The mRNA expression levels of INSR and IGF-1R were significantly reduced by $64 \%$ and $45 \%$ in siRNA transfected cells, respectively (Figure 2A). The protein expression level of $I G F-1 R$ was also reduced significantly (Figure 2B). However, the protein expression of INSR could not be examined due to the pig INSR antibody was not available.

Knockdown of INSR using siRNA decreased adipocyte differentiation showing by lower cytoplasm triglycerides content and the lipid droplets in mature adipocytes compared with the control (NC) (Figure 2C,D). Similar results were obtained from IGF-1R knockdown (Figure 2C,D). These results indicated that siRNA interference not only suppressed the expression of INSR and IGF-1R but also blocked hormone-induced adipocyte differentiation.

\subsection{The Differences of Insulin Signaling between Porcine i.m. Preadipocytes and s.c. Preadipocytes}

The expression levels of INSR and IGF-1R between i.m. and s.c. preadipcytes were compared to analyze the regional differences in adipogenesi. The mRNA expression levels of INSR and IGF-1R in i.m. preadipocytes were both significantly higher than those in s.c. preadipocytes (Figure 3A). Accordingly, the protein expression pattern of IGF-1R between i.m. and s.c. preadipocytes was consistent with the mRNA (Figure 3B). However, the protein expression of INSR could not be examined due to the pig INSR antibody was not available. 
A

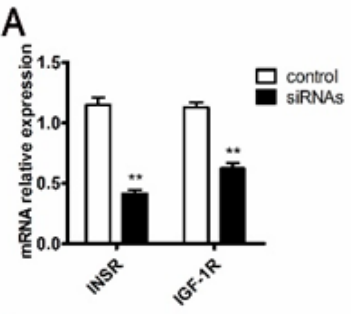

C

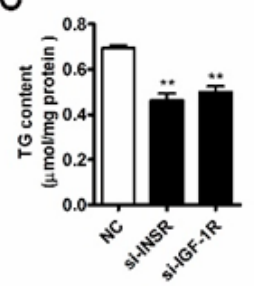

B

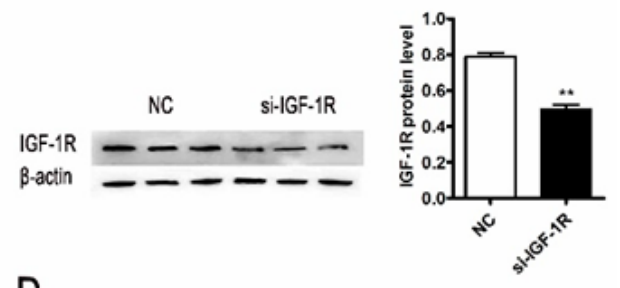

D

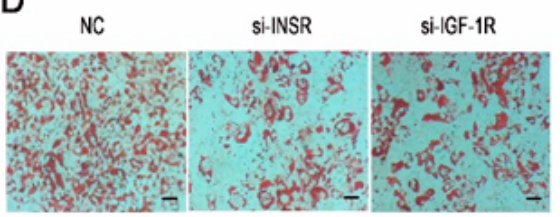

Figure 2. Downregulation of insulin receptor (INSR) or IGF-1 receptor (IGF-1R) by siRNA interference decreased porcine adipocyte differentiation. The siRNAs targeting INSR and IGF-1R (si-INSR, si-IGF-1R) were transfected into porcine s.c. preadipocytes. The INSR/IGF-1R mRNA (A) $(n=6)$ and protein levels (B) $(n=3)$ were verified after transfected with siNC and si-INSR/si-IGF-1R at 24 and $48 \mathrm{~h}$, respectively; Transfected s.c. preadipocytes were induced to differentiate for nine days; The intracellular accumulation was determined by cytoplasm triglyceride content $(C)(n=4)$ and Oil Red O staining (D) $(n=3)$. The mRNA expression differences were normalized to Ribosomal protein lateral stalk subunit P0 (RPLPO) mRNA level. The $\beta$-actin bands served as an internal control for protein loading. ${ }^{* *} p<0.01$, Scale bar $=200 \mu \mathrm{m}$.

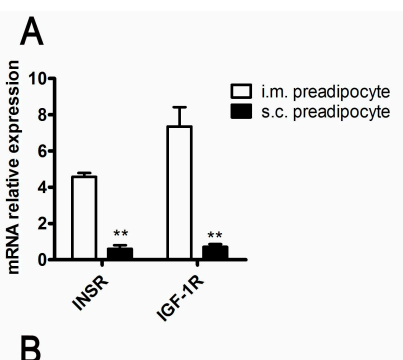

B

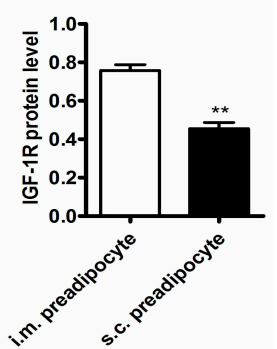

Figure 3. INSR and IGF-1R expression pattern in i.m. and s.c. preadipocytes. The comparison of INSR and IGF-1R mRNA expression levels in i.m. and s.c. preadipocytes $(\mathbf{A})(n=3)$; The protein expression level of IGF-1R in i.m. and s.c. preadipocytes was detected by Western blot and subsequently quantified. (B) $(n=3)$. The mRNA expression differences were normalized to RPLP0 mRNA level. The $\beta$-actin bands served as an internal control for protein loading. ${ }^{* *} p<0.01$.

\subsection{Identification and Its Effect on Adipocyte Differentiation}

The protein factors in the muscle conditional medium (MCM) were identified by label free quantitation (date not shown). There were so many kinds of proteins, including cytokines released from muscle tissue, adipose tissue and other endocrine organ. The s.c. preadipocytes were cultured with MCM at different concentrations to verify the effect of MCM on porcine adipocyte differentiation. 
During the differentiation, the s.c. preadipocytes were simply incubated in adipogenic medium supplemented with MCM at $0,10,40$, and $120 \mu \mathrm{g} \cdot \mathrm{mL}^{-1}$ total protein concentration. Nine days later, intracellular oil droplets were stained with Oil Red O. As indicated in Figure 4, MCM suppressed lipid accumulation with significant effects at $120 \mu \mathrm{g} \cdot \mathrm{mL}^{-1}$ total protein concentration. The inhibition effect of MCM on adipocyte differentiation was consistent with the development of i.m. preadipocytes in vivo. The amount of MCM at $120 \mu \mathrm{g} \cdot \mathrm{mL}^{-1}$ was used for further study.

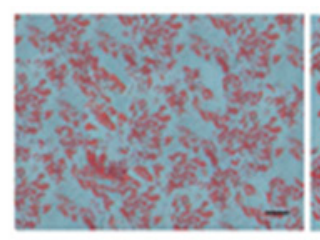

0

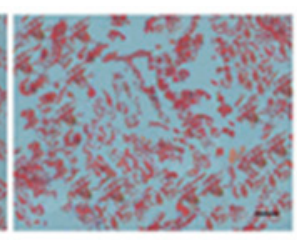

10

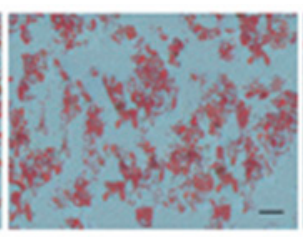

40

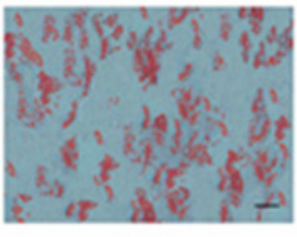

120

\section{Protein concentration $(\mu \mathrm{g} / \mathrm{mL})$}

Figure 4. The inhibitory effect of muscle conditional medium (MCM) on s.c. preadipocytes differentiation. Confluenced s.c. preadipocytes were incubated in adipogenic medium supplemented with an increasing amount of MCM at the indicated total protein concentration $(n=3)$. After nine days, adipocytes were staining with Oil Red O. Scale bar $=200 \mu \mathrm{m}$.

\subsection{Induced Apoptosis of Porcine Preadipocytes}

Porcine s.c. preadipocytes and s.c. adipocytes were respectively treated with MCM at $120 \mu \mathrm{g} \cdot \mathrm{mL}^{-1}$ total protein concentration for three days and then stained with fluorescein isothiocyanate (FITC)-labled annexin V (Annexin V-FITC and propidium iodine (PI) to detect cell apoptosis. Flow cytometric detection indicated that MCM induced significantly apoptosis in s.c. preadipocytes when compared with the control group (Figure 5A). The early and late apoptosis percentage was observed from $6.5 \%$ to $15.1 \%(p<0.01), 2.8 \%$ to $6.4 \%(p<0.05)$, respectively (Figure $5 \mathrm{~A})$. However, the apoptosis effects of MCM on s.c. adipocytes differed from that on s.c. preadipocytes. There was no difference in the apoptosis rate between control and the MCM-treated group (Figure 5B).
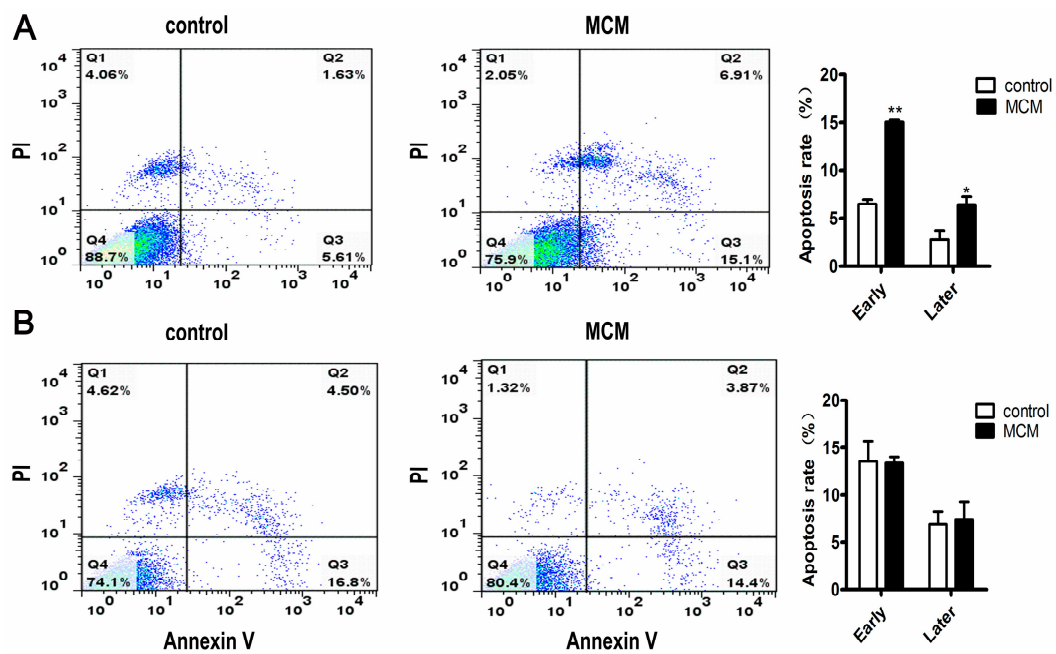

Figure 5. Flow cytometric detection and quantification of MCM-induced apoptosis in porcine s.c. preadipocytes and s.c. adipocytes. Porcine s.c. preadipocytes (A) and s.c. adipocytes (B) were cultured for three days with MCM versus physiological saline, and stained with fluorescein isothiocyanate (FITC)-labled annexin V (Annexin V-FITC) and propidium iodine (PI); then, the cell apoptosis was analyzed. $n=3,{ }^{*} p<0.05,{ }^{* *} p<0.01$, Scale bar $=200 \mu \mathrm{m}$. 


\subsection{Suppresses Adipocyte Differentiation during the Pre-Differentiation to the Middle Stage}

To elucidate the effective duration of activity of MCM during differentiation, s.c. preadipocytes were treated for three days with $\mathrm{MCM}$ at $120 \mu \mathrm{g} \cdot \mathrm{mL}^{-1}$ total protein concentration for four different time intervals: pre-differentiation (day -3 to 0 ), early (day 0 to 3), middle (day 4 to 6 ), and late (day 7 to 9) stages of differentiation.

The effect of MCM on adipocyte differentiation was different for the different stages. MCM significantly suppressed lipid accumulation with treatment at the pre-differentiation and early stage of differentiation, respectively, as indicated by less lipid droplets, decreased triglyceride content, and lower expression of adipocyte makers, peroxisome proliferator-activated receptor $\gamma(P P A R \gamma)$ and fatty acid binding protein (FABP4) (Figure 6A-C). While there was no difference in adipocyte differentiation with treatment at the middle stage of differentiation (Figure $6 \mathrm{~A}-\mathrm{C}$ ). When treated at the late stage of differentiation, $\mathrm{MCM}$ decreased triglyceride content and lower expression of adipocyte makers, $P P A R \gamma$ and FABP4 (Figure 6B,C). However, there was no difference in stained lipid droplets evaluated by Oil Red O staining (Figure 6A). These observations suggest that MCM inhibits adipocyte differentiation during pre-differentiation to middle stages of differentiation. Moreover, MCM reduces adipose tissue expansion by inhibiting lipid accumulation in adipocyte.

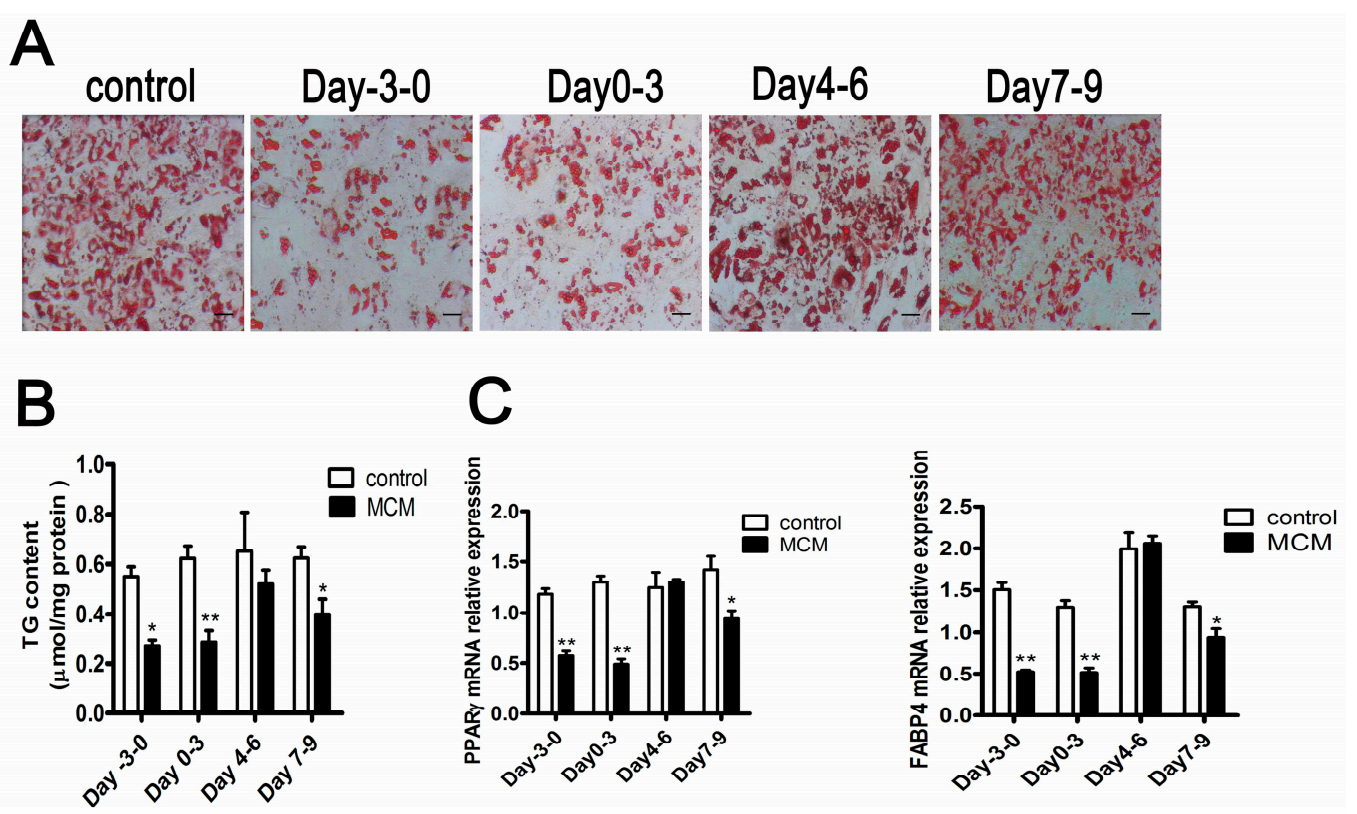

Figure 6. The effective duration of activity of MCM during adipocyte differentiation. s.c. preadipocytes were treated for three days with $\mathrm{MCM}$ at $120 \mu \mathrm{g} \mathrm{mL}^{-1}$ total protein concentration for four different time intervals: pre-differentiation (day -3 to 0 ), early (day 0 to 3 ), middle (day 4 to 6 ), and late (day 7 to 9) stages of differentiation. After nine days of differentiation, the effects of MCM on intracellular oil droplets (A) $(n=3)$, triglycerides content $(\mathbf{B})(n=3)$ and peroxisome proliferator-activated receptor $\gamma$ $(P P A R \gamma)$ and fatty acid binding protein $(F A B P 4)$ mRNA expression $(\mathbf{C})(n=6)$ were verified. The mRNA expression differences were normalized to RPLPO mRNA levels. Triglyceride content was normalized to protein content. ${ }^{*} p<0.05,{ }^{* *} p<0.01$, Scale bar $=200 \mu \mathrm{m}$.

\subsection{Increases INSR and IGF-1R Expression and Activates Insulin Signaling}

During the process of adipocyte differentiation treatment with $\mathrm{MCM}$, we focused on INSR/IGF-1R-IRS-1 expression in the insulin signaling cascade by qPCR and immunoblotting.

In the pre-differentiation stage of differentiation, MCM only led to a marked increase in IRS-1 mRNA expression $(p<0.01)$ (Figure 7A), while significantly increase in the protein expression of IGF-1R, IRS-1 and also the phosphorylation level of IRS-1(Try) $(p<0.01)$ (Figure 7B,C). During the 
different durations of differentiation, there was an increase in both the mRNA (Figure 7D-F) and protein expression (Figure 7G,H) of IGF-1R, IRS-1 and also the phosphorylation level of IRS-1 (Try) (Figure 7G,H) in MCM treated cells, with a significant increase mainly at the early and middle stages of differentiation. These data demonstrated that MCM increases INSR and IGF-1R expression and activates insulin signaling.

A
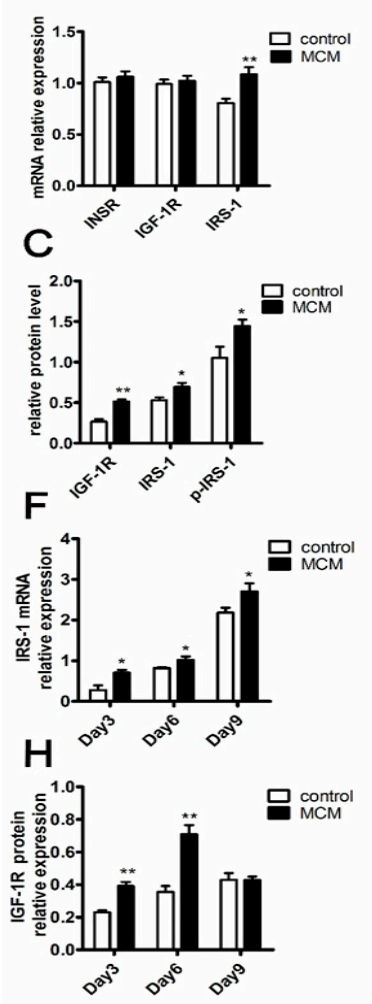

B

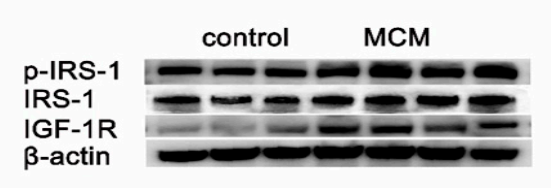

D

$\mathrm{E}$

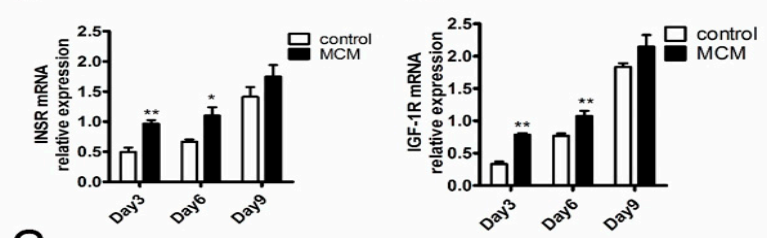

G
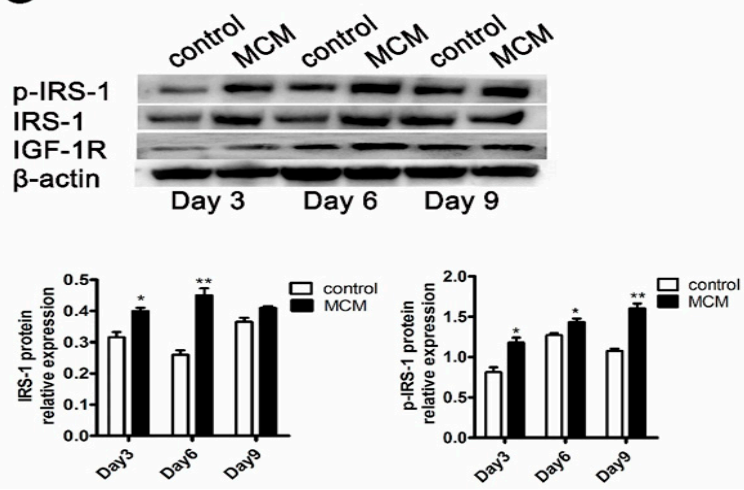

Figure 7. The genes expression patterns in the INSR/IGF-1R-IRS-1 signaling. The mRNA expression of INSR, IGF-1R and IRS-1 with MCM treatment at the pre-differentiation stage of adipocyte differentiation for three days (A) $(n=4)$; The protein abundance of IGF-1R, IRS-1, and $p$-IRS-1 with MCM treatment at pre-differentiation stage for three days (B) and protein quantified was shown (C) $(n=3$ for control,

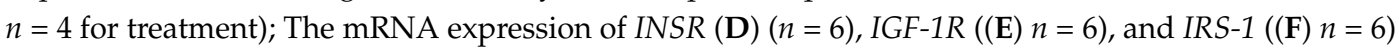
with MCM treatment for different durations; The protein abundance of IGF-1R, IRS-1, and $p-I R S-1$ with MCM treatment during different differentiation durations $(\mathbf{G})$ and protein quantified was shown ((H) $n=3)$; The mRNA expression differences were normalized to RPLPO mRNA level. The $\beta$-actin bands served as an internal control for protein loading, $p$-IRS-1 (Try) expression was normalized to IRS-1 expression. ${ }^{*} p<0.05,{ }^{* *} p<0.01$.

\subsection{Increases Adipocyte Lipolysis and Fatty Acid Oxidation}

The differences of lipid metabolism between control and MCM-treated s.c. adipocytes were analyzed by determining the content of glycerol release and the expression patterns of the genes involved in lipid metabolism. The lipolytic effects of MCM on porcine adipocyte were determined by treating s.c. adipocytes (day 9 after differentiation medium stimulation) with MCM for $24 \mathrm{~h}$. As shown in Figure 8A, MCM induced greater release of glycerol into the culture medium than control did. Additionally, MCM was added to the cells at day 6 of s.c. adipocyte differentiation for a total of three days of stimulation. Quantitative real-time PCR (qPCR) confirmed the reduction expression of genes encoding enzymes involved in fatty acid synthesis, including fatty acid synthase (FASN) and acetyl CoA carboxylase (ACC) (Figure $8 \mathrm{~B}$ ). Consistent with the increase of lipolysis, the mRNA expression of 
adipose triglyceride lipase (ATGL) and hormone sensitive lipase (HSL), which are the major lipases related to lipolysis, significantly increased in MCM-treated adipocytes (Figure 8B).

The expression of genes involves in fatty acid oxidation, including carnitine palmitoyl transferase $1 \alpha(C p t 1 \alpha)$, carnitine palmitoyl transferase $1 \beta(C p t 1 \beta)$ and peroxisome proliferator activated receptor $\alpha(P P A R \alpha)$, was also enhanced in MCM-treated adipocytes (Figure $8 \mathrm{C}$ ). Moreover, treatment of differentiated adipocytes with MCM resulted in a robust increase of peroxisome proliferator activated receptor- $1 \alpha$ (PGC-1 $\alpha$ ) mRNA levels together with an upregulation of the expression of additional key thermogenesis genes such as PR domain containing 16 (PRDM16) and cell death-inducing DFFA-like effector A (Cidea) (Figure 8D). Furthermore, the effects of MCM on mitochondrial biogenesis were also evaluated. Importantly, MCM induced a robust increase in mitochondrial content (Figure 8E). Compared to the control group (100\%), the mitochondrial DNA copy number in MCM-treated groups increased significantly $(184.5 \pm 22.5 \%, p<0.05)$ (Figure $8 \mathrm{E})$. The mRNA levels of genes implicated on mitochondrial biogenesis, including $P G C-1 \alpha$, nuclear respiratory factor-1 (NRF-1), mitochondrial transcription factor A (TFAM) were higher in MCM-treated adipocytes (Figure 8D-F). Taken together, the date suggested that MCM improved lipid metabolism by increasing adipocyte lipolysis and fat acid oxidation.
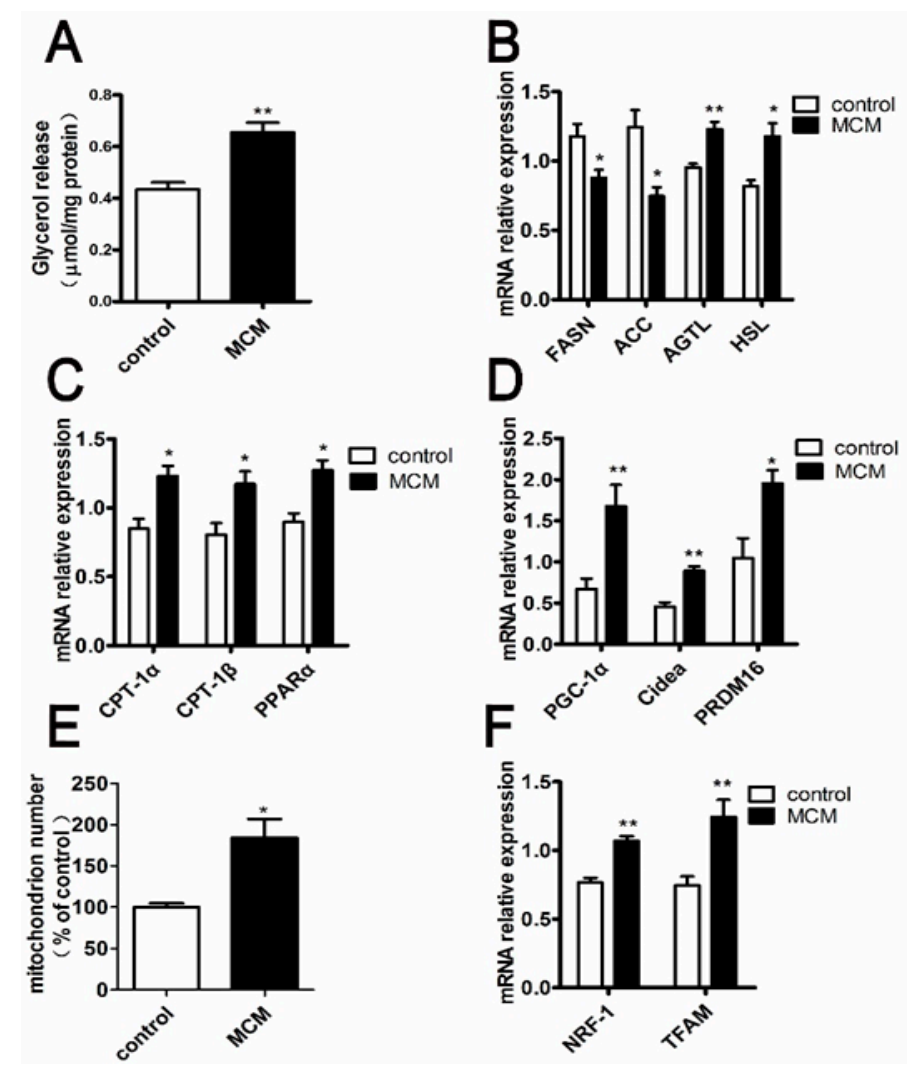

Figure 8. Effect of MCM on adipocyte lipolysis and fatty acid oxidation. Fully differentiated adipocytes were incubated with the Dulbecco's Modified Eagle's medium (DMEM) (no phenol red) with MCM for $24 \mathrm{~h}$ to verify the lipolytic effects of MCM. The glycerol levels in the culture medium were assayed (A); Adipocytes were cultured with MCM for three days. The expression levels of genes involved in lipolysis (ATGL and HSL) and fatty acid synthesis (FASN and ACC) (B) were detected by q PCR, respectively; Relative mRNA expression of genes responsible for fatty acid oxidation (PPAR, $\mathrm{Cpt}-1 \alpha$, and $C p t-1 \beta)(C)$ and thermogenesis (PGC-1 $\alpha, P R D M 16$, and Cidea) (D) was detected; Relative abundance of mitochondrial DNA (mtDNA) (E) and the genes responsible for mitochondrial biogenesis ( $P G C-1 \alpha$, TRFM, and NRF) (F) were detected. Dates were normalized to the mRNA levels of RPLP0. $n=6$, ${ }^{*} p<0.05,{ }^{* *} p<0.01$. 


\section{Discussion}

Regional variation in fat distribution affects economic values in pork production. Larger amounts of intramuscular fat can improve pork quality [19], whereas subcutaneous fat is considered to be one of the main sources of waste. The lipid metabolism and differentiation levels of i.m. adipocytes differed from that of s.c. adipocytes $[3,20,21]$. To study the molecular mechanism difference between i.m adipocytes development and s.c. adipocytes development, the i.m. and s.c. preadipocytes were isolated by the ceiling culture technique (Figure 1). These i.m. and s.c. preadipocytes originate from a homogeneous cell population derived from a single fraction of mature adipocytes. The cells proliferate extensively until they become confluent and differentiate into mature adipocytes upon treatment with insulin, dexamethasone (Dex) and 3-isobutyl-1-methylxanthine (IBMX) (Figure 1B). The cells proliferate more effectively compared to the stromal-vascular fraction isolated from traditionally collagenase technique [22].

The variety biology effects of insulin in adipocytes are mediated by insulin receptor (INSR) as well as IGF-1 receptor (IGF-1R), both of them belong to the family of receptor tyrosine kinases [23]. Importantly, the two receptors activate common intracellular pathways by using similar mechanisms [24]. However, one receptor cannot functionally compensate when the other one is absent [25]. Thus, the adipogenesis role of INSR and IGF-1R were verified in this study. Downregulation of INSR and IGF-1R, respectively, lead to the reduction of adipocyte differentiation (Figure 2), which consisted of previous results in mice adipogenesis research [13,26,27]. In vivo study, mice with the fat-specific insulin receptors and/or IGF-1 receptors knockout have reduced fat mass [26,27]. Moreover, decrease in expression of 3T3-L1 preadipocyte insulin receptor impaired adipocyte differentiation [13]. All of these findings demonstrated that INSR and IGF-1R signaling are essential molecules for adipogenesis.

The regional differences of INSR and IGF-1R expression were compared between i.m. preadipocyte and s.c. preadipocyte. The mRNA expression level of INSR and IGF-1R in i.m. preadipocytes was higher than that in s.c. preadipocytes (Figure 3A). The similar gene expression pattern was seen in protein level (Figure 3B). Compared with subcutaneous adipose tissue, the higher expression level of $I N S R$ and IGF-1R in intramuscular adipose tissue did not render more fat in this regional, suggesting that the extracellular micro-environment of the i.m. preadipocytes has an important influence.

Adipose tissue is a very important organ for secretion of many endocrine and paracrine factors, collectively referred to adipokines [28,29]. Accordingly, the skeletal muscle has also been identified as an endocrine organ that produces and releases cytokines, which were named "myokines" [30-32]. These cytokines influence adipose tissue development in coordination with endocrine hormones via endocrine, paracrine, and autocrine signals [28,33,34]. Due to the particular location in close vicinity with muscle fibers, the myokines have more influence on i.m. adipocytes metabolism than s.c. adipocytes. To approximate the effects of extracellular micro-environment on i.m. preadipocyte development in vivo, the s.c. preadipocytes were cultured with MCM. In contrast to one or more purified hormones or cytokines, MCM represents the specific combination of factors that exist within the tissue micro-environment.

During the process of adipocyte differentiation, lipid accumulation reduced significantly when s.c. preadipocytes were treated with MCM at pre-differentiation and early stages of differentiation, while the expression levels of INSR and IGF-1R were increased (Figures 6 and 7). The suppression effects of MCM on adipgenesis were supported by previous studies that myocytes suppressed adipocyte differentiation in pig and mouse research [35,36]. Similarly, the accumulation of INSR and IGF-1R, resulting from MCM treatment, did not render cells more adipogenic. The impaired differentiation was partially due to the induction apoptosis effects of MCM on porcine s.c. preadipocytes (Figure 5). Many myokines, such as interleukin-6, induced lipolysis and fatty acid oxidation in adipocytes [30]. The present study revealed that MCM induced adipocytes lipolysis and fatty acid oxidation in mitochondria (Figure 8). Importantly, the expression levels of genes involved in lipolysis and fatty acid oxidation were increased, while fatty acid synthesis related genes, including FASN and ACC, were 
decreased. These date may relate to the reduction of triglyceride content in adipocytes when treated s.c. adipocytes with MCM at the late stage of differentiation (Figure 6B,C).

To further verify the fatty acid oxidation in mitochondria, we evaluated the effects of $\mathrm{MCM}$ on mitochondrial biogenesis. Consistent with fatty acid oxidation, the mitochondrial DNA copy number and expression levels of marker genes responsible for mitochondrial biogenesis were increased significantly (Figure 8E,F). The date indicated that MCM could upregulate the mitochondrial biogenesis in s.c. adipocytes.

There are two different types of adipose tissue, namely white adipose tissue (WAT) and brown adipose tissue (BAT). The main function of WAT is to store energy, whereas the BAT is specialized to dissipate energy as heat. Generally, brown adipocytes are predominantly located in BAT. It can also emerge among WAT through the browning process. Cold exposure, hormones, enzymes, transcription factors, and microRNAs have recently been shown to drive the so-called browning process [37,38]. PGC-1 $\alpha$ and PRDM16 were required for the switch from WAT to beige cells [39]. Moreover, PGC-1 $\alpha$ and Cidea were usually regarded as the brown adipogenic marker in the regulation of brown and beige fat $[40,41]$. In the current study, MCM indeed increased the major thermogenesis genes expression, concluding PGC-1 $\alpha$, Cidea and PRDM16, in s.c. adipocytes (Figure 8D). Furthermore, Figure 8C,E revealed a significantly higher fatty acid oxidation capacity and mitochondrial copy number in MCM-treated s.c. adipocytes than that in control cells. These findings indicated that MCM might promote the white to brite trans-differentiation.

\section{Materials and Methods}

\subsection{Porcine Preadipocytes Isolation, Culture and Identification}

All the animals used in this experiments were performed according to "The Guidelines for the Care of Laboratory Animals" enacted by the Ministry of Science and Technology of People's Republic of China. Three-days-old Suhuai pigs from Animal Husbandry Group in Chang Shu of China were killed via intraperitoneal injection of pentobarbital sodium $(50 \mathrm{mg} / \mathrm{kg}$ body weight) followed by exsanguinations. Dedifferentiated intramuscular (i.m.) and subcutaneous (s.c.) preadipocytes were isolated using the ceiling culture method. Briefly longissimus dorsi muscle tissue and subcutaneous adipose tissue were sampled, respectively, and cut into pieces followed by digested with $1 \mathrm{mg} / \mathrm{mL}$ type I collagenase for about $2 \mathrm{~h}$ at $37^{\circ} \mathrm{C}$ in a shaking water bath. Then, the two kinds of digested tissues were filtered with $75-\mu \mathrm{m}$ steel mesh, and cells were washed twice with DMEM by centrifugation at $200 \times g$ for $10 \mathrm{~min}$. The mature adipocytes were collected from the floating layer and placed into $\mathrm{T} 25-\mathrm{cm}^{2}$ flasks completely filled with DMEM containing 10\% fetal bovine serum (FBS). Flasks containing mature adipocytes were inverted and then incubated in a $5 \% \mathrm{CO}_{2}$ incubator at $37^{\circ} \mathrm{C}$ for about 10 days. Mature adipocytes were able to dedifferentiate into proliferative-competent preadipocytes. The excess cell culture media was removed and the culture flask was turned upside-down. The medium was changed every 2 days.

The isolated preadipocytes were cultured in Dulbecco's Modified Eagle's medium (DMEM) containing $10 \% \mathrm{FBS}$ and $1 \%$ penicillin-streptomycin at $37^{\circ} \mathrm{C}$ in $5 \% \mathrm{CO}_{2}$. When reaching confluence, the preadipocytes were induced to differentiate into adipocytes by culturing in differentiation medium containing 10\% FBS-DMEM supplemented with dexamethasone $(0.25 \mathrm{mM})$, IBMX $(1 \mathrm{mM})$, and insulin $\left(5 \mu \mathrm{g} \mathrm{mL}^{-1}\right)$ (DMI) (Sigma-Aldrich, Shanghai, China). After 3 days, the medium was replaced with 10\% FBS-DMEM until day 9. Fresh new medium was changed every 2 days.

The i.m. and s.c. preadipocytes were seeded in 12-well plates to demonstrate that the isolated cells were preadipocytes with high purity. Briefly, the two types of cells were fixed in ice-cold $4 \%$ paraformaldehyde for about $10 \mathrm{~min}$, then permeabilized with $1 \%$ Triton X-100 for $10 \mathrm{~min}$, and incubated with $5 \%$ bovine serum albumin (BSA) for $1 \mathrm{~h}$ at room temperature. After washing 3 times with PBS later ( 5 min each), the cells were incubated with the primary antibodies of rabbit anti-porcine Pref-1 (DLK) (Santa Cruz Biotech Inc., Santa Cruz, CA, USA; sc-25437, 1:100 dilution) overnight at $4{ }^{\circ} \mathrm{C}$. 
The cells were then washed twice with PBS and incubated with the secondary antibody of kFluor594 labeled goat anti-rabbit IgG antibodies (Keygen Biotech, Nanjing, China; kgif008, 1:500 dilution) for $1 \mathrm{~h}$ at room temperature in the dark. The verified images were obtained using a Confocal Laser Scanning Microscope (Carl Zeiss LSM700, Oberkochen, Germany).

\subsection{Oil Red O Staining and Triglyceride Measurements}

To quantify lipid accumulation, Oil Red O staining and cytoplasm triglyceride content were performed on day 9 of adipocyte differentiation. Briefly, the adipocytes were washed twice with phosphate-buffered saline (PBS), fixed with 10\% phosphate-buffered formalin for $15 \mathrm{~min}$, stained with Oil Red O working solution for $30 \mathrm{~min}$ at room temperature, and then washed for $20 \mathrm{~s}$ with $60 \%$ isopropanol to remove the excess stain. The stained lipid droplets within adipocytes were visualized by Leica Inverted Fluorescence Microscope (Wetzlar, Germany).

Measurement of intracellular triglyceride levels were performed by Triglyceride assay kit (Applygen Technologies Inc., Beijing, China) according to the manufacturer's instructions. Triglyceride levels were normalized to protein concentration. Protein content was measured by a BCA Protein Assay Kit (Beyotime, Shanghai, China) according to the manufacturer's instructions.

\section{3. mRNA Expression Analysis}

The total RNA from cells was extracted with Trizol reagent (Invitrogen, Carlsbad, CA, USA) according to the manufacturer's instructions. The mRNA expression levels of related genes were detected by Synergy brands (SYBR) Green Dye quantitative real-time PCR (qPCR) (Takara, Dalian, China) with the Applied Biosystems StepOneTM detection System (Applied Biosystems, Foster City, CA, USA). Samples were run in triplicate for each experiment and were normalized to Ribosomal protein lateral stalk subunit P0 (RPLPO) to determine relative expression level. Primer sets used in this study were provided in Table 1.

Table 1. Primer sequences of related gene for qPCR.

\begin{tabular}{|c|c|c|c|}
\hline Gene & Primer Sequence $\left(5^{\prime}-3^{\prime}\right)$ & Product Size (bp) & GeneBank No. \\
\hline INSR & $\begin{array}{l}\text { F: GTGTTGTGATTGGAAGCATTTAT } \\
\text { R: CATCGCTGGCACTGAGGTA }\end{array}$ & 108 & XM_021083940.1 \\
\hline$F A B P 4$ & $\begin{array}{l}\text { F: GCCAAACCCAACCTGATCAT } \\
\text { R: TCCCACTTCTGCACCTGTAC }\end{array}$ & 188 & NM_001002817.1 \\
\hline PPAR $\gamma$ & $\begin{array}{l}\text { F: TTGCTGTGAAGTTCAACGCA } \\
\text { R: GTGGTTCAACTTGAGCTGCA }\end{array}$ & 167 & NM_214379.1 \\
\hline$I G F-1 R$ & $\begin{array}{l}\text { F: CAACCTCCGGCCTTTTACTTT } \\
\text { R: CAGGAATGTCATCTGCTCCTTCT }\end{array}$ & 134 & NM_214172.1 \\
\hline RPLPO & $\begin{array}{l}\text { F: TCCAGGCTTTAGGCATCACC } \\
\text { R: GGCTCCCACTTTGTCTCCAG }\end{array}$ & 95 & NM_001098598.1 \\
\hline FASN & $\begin{array}{c}\text { F: GCTTGTCCTGGGAAGAGTGTA } \\
\text { R: AGGAACTCGGACATAGCGG }\end{array}$ & 114 & NM_001099930.1 \\
\hline$A C C$ & $\begin{array}{l}\text { F: CGCTTCATAATTGGTTCTGTG } \\
\text { R: GCTAGAAATCCCCAAGTCAGA }\end{array}$ & 147 & NM_001114269.1 \\
\hline ATGL & $\begin{array}{l}\text { F: GCGAAAATGTCATCATAACC } \\
\text { R: ATGGTGCTCTTGAGTTCGT }\end{array}$ & 175 & NM_001098605.1 \\
\hline$H S L$ & $\begin{array}{l}\text { F: GCCCGAGACGAGATTAG } \\
\text { R: ATGAAGGGATTCTTGACG }\end{array}$ & 143 & NM_214315.2 \\
\hline$C P T-1 \alpha$ & $\begin{array}{l}\text { F: AACCTTCTGGCGGACGACG } \\
\text { R: GCAGGAACGCACGGTCTCA }\end{array}$ & 213 & NM_001129805.1 \\
\hline$C P T-1 \beta$ & $\begin{array}{l}\text { F:ACTGTCTGGGCAAACCAAAC } \\
\text { R: CTTCTTGATGAGGCCTTTGC }\end{array}$ & 176 & NM_001007191.1 \\
\hline
\end{tabular}


Table 1. Cont.

\begin{tabular}{cccc}
\hline Gene & Primer Sequence $\left(\mathbf{5}^{\prime}-\mathbf{3}^{\prime}\right)$ & Product Size (bp) & GeneBank No. \\
\hline \multirow{2}{*}{$P$ PAR $\alpha$} & $\begin{array}{c}\text { F:CAGCCTCCAGCCCCTCGTC } \\
\text { R: GCGGTCTCGGCATCTTCTAGG } \\
\text { F: CTGTGGATGAAGACGGATTG } \\
\text { R: GTCAGGCATGGAGGAAGGA }\end{array}$ & 382 & NM_001044526.1 \\
\hline \multirow{2}{*}{ Cidea } & $\begin{array}{l}\text { F: TTCCGAGTTTCCAACCACAA } \\
\text { R: CGATAACCAGGGCATCCAG }\end{array}$ & 92 & NM_213963.2 \\
\hline \multirow{2}{*}{$P R D M 16$} & $\begin{array}{c}\text { F: CCACAAGTCCTACACGCAGTTCTC } \\
\text { R: GTTGAGGGACGAGGTAGTGCTGA }\end{array}$ & 121 & NM_001112696 \\
\hline \multirow{2}{*}{ TFAM } & $\begin{array}{c}\text { F:GGTCCATCACAGGTAAAGCTGAA } \\
\text { R:ATAAGATCGTTTCGCCCAACTTC }\end{array}$ & 167 & XM_021095209.1 \\
\hline \multirow{2}{*}{ NRF-1 } & $\begin{array}{c}\text { F: CAACAGGAAAGAAACGGAAAC } \\
\text { R: GAGGGTGAGATACAAAGGACAAT }\end{array}$ & 157 & NM_001130211.1 \\
\hline
\end{tabular}

\subsection{Isolation and Identification of Interstitial Fluid from Skeletal Muscle by a Centrifugation Method}

Longissimus dorsi muscle ( $20 \mathrm{~g})$ were excised, flushed with saline to remove the possible blood and intracellular fluid from the surface, blotted gently with tissue paper to remove excess saline, and transferred to $50 \mathrm{~mL}$ centrifuge TUBES used for isolation of muscle interstitial fluid [42]. To reduce the risk of muscle cell compression, the centrifugation speed was $1000 \times g$ for $10 \mathrm{~min}$. The interstitial fluid accumulated at the bottom of tube was collected, filtered through a $0.2 \mu \mathrm{m}$ filter, analyzed for protein content, stored at $-80{ }^{\circ} \mathrm{C}$ and designed as muscle conditioned medium (MCM). In this study, the overall process was carried out at $4{ }^{\circ} \mathrm{C}$. The protein factors in the muscle conditional medium $(\mathrm{MCM})$ were identified by label free quantitation.

\section{5. siRNAs Trasfection}

Porcine s.c. preadipocytes were transfected with siRNA oligos using lipofectamine 2000 transfection reagent (Invitrogen, Carlsbad, CA, USA) following the manufacturer's manual. The cell were $80 \%$ confluence during transfection. The siRNA/lipofectamine 2000 complexes were prepared in opti-MEM media and added dropwise to the cells.

\subsection{Western Blotting Analyses}

The cellular total protein was extracted via addition of RIPA lysis buffer (Beyotime, Shanghai, China) to the cell pellets on ice for $30 \mathrm{~min}$, and protein concentration was measured with a BCA protein quantification kit (Beyotime, Shanghai, China). The protein samples $(50 \mu \mathrm{g})$ were separated by sodium dodecyl sulphate-polyacrylamide gel electrophoresis (SDS-PAGE), transferred to polyvinylidene fluoride membranes (PVDF) and blotted with specific primary antibodies for IGF-1R (Santa Cruz Biotech Inc., sc-713, 1:200 dilution), IRS-1 (Santa Cruz Biotech Inc, sc-560, 1:200 dilution), p-IRS-1 (Tyr 632) (Santa Cruz Biotech Inc., sc-17196, 1:200 dilution ), and $\beta$-actin (Santa Cruz Biotech Inc., sc-47778, 1:1000 dilution). After incubation with horseradish-peroxidase-conjugated (HPR) secondary antibody for $2 \mathrm{~h}$ at room temperature, the protein expression was visualized with an enhanced chemiluminescent reagent (ECL, Thermo Fisher Scientific, City, MA, USA) by Versa DosTM 4000 MP (Bio-Rad Laboratories, Munich, Germany).

\subsection{Glycerol Release}

The s.c. adipocytes (day 9 after differentiation medium stimulation) were washed once with PBS gently and then incubated with DMEM (no phenol red and FBS) (Wisent Biotechnology, Nanjing, China) containing with or without MCM for $24 \mathrm{~h}$. The culture medium of samples were collected and glycerol levels in the media were assayed using a glycerol kit (Applygen Technologies Inc., Beijing, China) according to the manufacturer' instructions. 


\subsection{Analysis of Mitochondrial DNA Content}

The mitochondrial DNA (mtDNA) content was assessed using a modification of the quantitative real-time PCR (qPCR) as described previously [43,44]. Briefly, the total DNA was extracted from s.c. adipocytes using DNA extraction kit (Jiancheng Bioengineering Institute, Nanjing, China; $\mathrm{d} 1700-50)$. The relative amount of mtDNA was quantified by comparison of a mitochondrial target, the cytochrome c oxidase subunit II (COX2) with a nuclear target, ribosomal protein lateral stalk subunit P0 (RPLP0). Quantitative real-time PCR was performed using Applied Biosystems StepOneTM detection System (Applied Biosystems, Foster City, CA, USA). For quantification, reference curves that were serial dilutions of a standard DNA were used. Primer sequences of COX2 and RPLP0 for qPCR was 5'-AGGACGACTAAACCAAACA-3' , 5'-AATGGGACAAGTTCAAGTA-3 ${ }^{\prime}$ and 5'-ATGGCAGCATCTACAACC-C-3', 5'-AGACAAAGCCAGGACCCAC-3', respectively. A ratio between COX2 and RPLP0 was calculated (COX2/RPLP0), which was used as the mtDNA content in the present study. Each sample was measured at least in triplicate, and mean values were calculated.

\subsection{Ethics Statement}

All experiments were performed in accordance with the guidelines of the regional Animal Ethics Committee and were approved by the Institutional Animal Care and Use Committee of Nanjing Agricultural University (NJAU-CAST-2014-179, 1 January 1978).

\subsection{Statistical Analysis}

Results are expressed as the mean \pm SEM. Graphpad Prism software (Graphpad Prism 5 V 5.01, GraphPad Prism Software Inc., San Diego, CA, USA) was used for all analyses. The comparison performed using Student's $t$-test or one-way NAOVA. Minimal level of significance was set at $p<0.05$. All experiments were performed a minimum of three times.

\section{Conclusions}

INSR and IGF-1R signaling are essential molecules for porcine adipogenesis. MCM induced an apoptotic effect on preadipocytes. Moreover, MCM inhibited adipocyte differentiation at pre-differentiation and early stages of differentiation, while the expression levels of INSR and IGF-1R were increased. The lipid metabolism was improved via induction of adipocytes lipolysis, fatty acid oxidation and expression levels of key thermogenesis genes by MCM. In conclusion, the effects of MCM on lipid metabolism could approximate the development of i.m. preadipocytes in vivo.

Acknowledgments: This work was supported by the National Key Technology Support Program (Grant No. 2015BAD03B01) and the National Natural Science Foundation of China (Grant No. 31272423).

Author Contributions: Jie Chen designed the study in the manuscript; Haiyin Han participated in the design and performed the experiments in the manuscript; Wei Wei and Weiwei Chu analyzed the experiments and prepared the figures; Kaiqing Liu, Ye Tian and Zaohang Jiang wrote the final version of the manuscript; All authors reviewed the results and approved the final version of the manuscript.

Conflicts of Interest: The authors declare no conflict of interest. 


\section{Abbreviations}

$\begin{array}{ll}\text { INSR } & \text { Insulin receptor } \\ \text { IGF-1R } & \text { Insulin-like growth factor 1 receptor } \\ \text { MCM } & \text { Muscle conditional cultured medium } \\ \text { IMF } & \text { Intramuscular fat } \\ \text { DMEM } & \text { Dulbecco's Modified Eagle's medium } \\ \text { FBS } & \text { Fetal bovine serum } \\ \text { SDS-PAGE } & \text { Sodium dodecyl sulphate-polyacrylamide gel electrophoresis } \\ \text { PVDF } & \text { Polyvinylidene fluoride membranes } \\ \text { mtDNA } & \text { Mitochondrial DNA } \\ \text { COX2 } & \text { Cytochrome c oxidase subunit II } \\ \text { RPLP0 } & \text { Ribosomal protein lateral stalk subunit P0 } \\ \text { PI } & \text { Propidium iodine } \\ \text { NRF-1 } & \text { Nuclear respiratory factor-1 } \\ \text { TFAM } & \text { Mitochondrial transcription factor A } \\ \text { WAT } & \text { White adipose tissue } \\ \text { BAT } & \text { Brown adipose tissue }\end{array}$

\section{References}

1. Gardan, D.; Gondret, F.; Louveau, I. Lipid metabolism and secretory function of porcine intramuscular adipocytes compared with subcutaneous and perirenal adipocytes. Am. J. Physiol. Endocrinol. Metab. 2006, 291, E372-E380. [CrossRef] [PubMed]

2. Smith, S.B.; Crouse, J.D. Relative contributions of acetate, lactate and glucose to lipogenesis in bovine, intramuscular and subcutaneous adipose tissue. J. Nutr. 1984, 114, 792-800. [PubMed]

3. Lee, Y.B.; Kauffman, R.G. Cellularity and lipogenic enzyme activities of porcine intramuscular adipose tissue. J. Anim. Sci. 1974, 38, 538-544. [CrossRef] [PubMed]

4. Spiegelman, B.M.; Flier, J.S. Adipogenesis and obesity: Rounding out the big picture. Cell 1996, 87, 377-389. [CrossRef]

5. Suryawan, A.; Swanson, L.V.; Hu, C.Y. Insulin and hydrocortisone, but not triiodothyronine, are required for the differentiation of pig preadipocytes in primary culture. J. Anim. Sci. 1997, 75, 105-111. [CrossRef] [PubMed]

6. Gagnon, A.; Sorisky, A. The effect of glucose concentration on insulin-induced 3T3-L1 adipose cell differentiation. Obes. Res. 1998, 6, 157-163. [CrossRef] [PubMed]

7. Klemm, D.J.; Leitner, J.W.; Watson, P.; Nesterova, A.; Reusch, J.E.; Goalstone, M.L.; Draznin, B. Insulin-induced adipocyte differentiation. Activation of CREB rescues adipogenesis from the arrest caused by inhibition of prenylation. J. Biol. Chem. 2001, 276, 28430-28435. [CrossRef] [PubMed]

8. Reed, B.C.; Lane, M.D. Insulin receptor synthesis and turnover in differentiating 3T3-L1 preadipocytes. Proc. Natl. Acad. Sci. USA 1980, 77, 285-289. [CrossRef] [PubMed]

9. Klaman, L.D.; Boss, O.; Peroni, O.D.; Kim, J.K.; Martino, J.L.; Zabolotny, J.M.; Moghal, N.; Lubkin, M.; Kim, Y.B.; Sharpe, A.H.; et al. Increased energy expenditure, decreased adiposity, and tissue-specific insulin sensitivity in protein-tyrosine phosphatase 1B-deficient mice. Mol. Cell Biol. 2000, 20, 5479-5489. [CrossRef] [PubMed]

10. Smith, P.J.; Wise, L.S.; Berkowitz, R.; Wan, C.; Rubin, C.S. Insulin-like growth factor-I is an essential regulator of the differentiation of 3T3-L1 adipocytes. J. Biol. Chem. 1988, 263, 9402-9408. [PubMed]

11. Miki, H.; Yamauchi, T.; Suzuki, R.; Komeda, K.; Tsuchida, A.; Kubota, N.; Terauchi, Y.; Kamon, J.; Kaburagi, Y.; Matsui, J.; et al. Essential role of insulin receptor substrate 1 (IRS-1) and IRS-2 in adipocyte differentiation. Mol. Cell Biol. 2001, 21, 2521-2532. [CrossRef] [PubMed]

12. Myers, M.G., Jr.; White, M.F. New frontiers in insulin receptor substrate signaling. Trends Endocrinol. Metab. 1995, 6, 209-215. [CrossRef]

13. Accili, D.; Taylor, S.I. Targeted inactivation of the insulin receptor gene in mouse 3T3-L1 fibroblasts via homologous recombination. Proc. Natl. Acad. Sci. USA 1991, 88, 4708-4712. [CrossRef] [PubMed] 
14. Hirai, S.; Matsumoto, H.; Hino, N.; Kawachi, H.; Matsui, T.; Yano, H. Myostatin inhibits differentiation of bovine preadipocyte. Domest. Anim. Endocrinol. 2007, 32, 1-14. [CrossRef] [PubMed]

15. Quinn, L.S. Interleukin-15: A muscle-derived cytokine regulating fat-to-lean body composition. J. Anim. Sci. 2008, 86, E75-E83. [CrossRef] [PubMed]

16. Huh, J.Y.; Dincer, F.; Mesfum, E.; Mantzoros, C.S. Irisin stimulates muscle growth-related genes and regulates adipocyte differentiation and metabolism in humans. Int. J. Obes. 2014, 38, 1538-1544. [CrossRef] [PubMed]

17. Zhou, X.; Li, D.; Yin, J.; Ni, J.; Dong, B.; Zhang, J.; Du, M. CLA differently regulates adipogenesis in stromal vascular cells from porcine subcutaneous adipose and skeletal muscle. J. Lipid Res. 2007, 48, 1701-1709. [CrossRef] [PubMed]

18. Shan, T.; Liu, W.; Kuang, S. Fatty acid binding protein 4 expression marks a population of adipocyte progenitors in white and brown adipose tissues. FASEB J. 2013, 27, 277-287. [CrossRef] [PubMed]

19. Wood, J.D.; Enser, M.; Fisher, A.V.; Nute, G.R.; Richardson, R.I.; Sheard, P.R. Manipulating meat quality and composition. Proc. Nutr. Soc. 1999, 58, 363-370. [CrossRef] [PubMed]

20. Wang, S.; Zhou, G.; Shu, G.; Wang, L.; Zhu, X.; Gao, P.; Xi, Q.; Zhang, Y.; Yuan, L.; Jiang, Q. Glucose utilization, lipid metabolism and BMP-Smad signaling pathway of porcine intramuscular preadipocytes compared with subcutaneous preadipocytes. Cell. Physiol. Biochem. 2013, 31, 981-996. [CrossRef] [PubMed]

21. Wan, R.; Du, J.; Ren, L.; Meng, Q. Selective adipogenic effects of propionate on bovine intramuscular and subcutaneous preadipocytes. Meat Sci. 2009, 82, 372-378. [CrossRef] [PubMed]

22. Nobusue, H.; Kano, K. Establishment and characteristics of porcine preadipocyte cell lines derived from mature adipocytes. J. Cell Biochem. 2010, 109, 542-552. [CrossRef] [PubMed]

23. Ullrich, A.; Schlessinger, J. Signal transduction by receptors with tyrosine kinase activity. Cell 1990, 61, 203-212. [CrossRef]

24. Entingh-Pearsall, A.; Kahn, C.R. Differential roles of the insulin and insulin-like growth factor-I (IGF-I) receptors in response to insulin and IGF-I. J. Biol. Chem. 2004, 279, 38016-38024. [CrossRef] [PubMed]

25. Bluher, S.; Kratzsch, J.; Kiess, W. Insulin-like growth factor I, growth hormone and insulin in white adipose tissue. Best. Pract. Res. Clin. Endocrinol. Metab. 2005, 19, 577-587. [CrossRef] [PubMed]

26. Boucher, J.; Mori, M.A.; Lee, K.Y.; Smyth, G.; Liew, C.W.; Macotela, Y.; Rourk, M.; Bluher, M.; Russell, S.J.; Kahn, C.R. Impaired thermogenesis and adipose tissue development in mice with fat-specific disruption of insulin and IGF-1 signalling. Nat. Commun. 2012, 3, 902. [CrossRef] [PubMed]

27. Bluher, M.; Kahn, B.B.; Kahn, C.R. Extended longevity in mice lacking the insulin receptor in adipose tissue. Science 2003, 299, 572-574. [CrossRef] [PubMed]

28. Avram, A.S.; Avram, M.M.; James, W.D. Subcutaneous fat in normal and diseased states: 2. Anatomy and physiology of white and brown adipose tissue. J. Am. Acad. Dermatol. 2005, 53, 671-683. [CrossRef] [PubMed]

29. Avram, M.M.; Avram, A.S.; James, W.D. Subcutaneous fat in normal and diseased states 3. Adipogenesis: From stem cell to fat cell. J. Am. Acad. Dermatol. 2007, 56, 472-492. [CrossRef] [PubMed]

30. Pedersen, B.K.; Febbraio, M.A. Muscle as an endocrine organ: Focus on muscle-derived interleukin-6. Physiol. Rev. 2008, 88, 1379-1406. [CrossRef] [PubMed]

31. Pedersen, B.K.; Steensberg, A.; Fischer, C.; Keller, C.; Keller, P.; Plomgaard, P.; Febbraio, M.; Saltin, B. Searching for the exercise factor: Is IL-6 a candidate? J. Muscle Res. Cell Motil. 2003, 24, 113-119. [CrossRef] [PubMed]

32. Pedersen, B.K.; Akerström, T.C.; Nielsen, A.R.; Fischer, C.P. Role of myokines in exercise and metabolism. J. Appl. Physiol. 2007, 103, 1093-1098. [CrossRef] [PubMed]

33. Halberg, N.; Wernstedt-Asterholm, I.; Scherer, P.E. The adipocyte as an endocrine cell. Endocrinol. Metab. Clin. N. Am. 2008, 37, 753-768. [CrossRef] [PubMed]

34. Pedersen, B.K.; Febbraio, M.A. Muscles, exercise and obesity: Skeletal muscle as a secretory organ. Nat. Rev. Endocrinol. 2012, 8, 457-465. [CrossRef] [PubMed]

35. Dietze, D.; Koenen, M.; Röhrig, K.; Horikoshi, H.; Hauner, H.; Eckel, J. Impairment of insulin signaling in human skeletal muscle cells by co-culture with human adipocytes. Diabetes 2002, 51, 2369-2376. [CrossRef] [PubMed]

36. Chu, W.; Wei, W.; Yu, S.; Han, H.; Shi, X.; Sun, W.; Gao, Y.; Zhang, L.; Chen, J. C2C12 myotubes inhibit the proliferation and differentiation of 3T3-L1 preadipocytes by reducing the expression of glucocorticoid receptor gene. Biochem. Biophys. Res. Commun. 2016, 472, 68-74. [CrossRef] [PubMed] 
37. Loncar, D. Convertible adipose tissue in mice. Cell Tissue Res. 1991, 266, 149-161. [CrossRef] [PubMed]

38. Wu, J.; Cohen, P.; Spiegelman, B.M. Adaptive thermogenesis in adipocytes: Is beige the new brown? Genes Dev. 2013, 27, 234-250. [CrossRef] [PubMed]

39. Cohen, P.; Levy, J.D.; Zhang, Y.; Frontini, A.; Kolodin, D.P.; Svensson, K.J.; Lo, J.C.; Zeng, X.; Ye, L.; Khandekar, M.J.; et al. Ablation of PRDM16 and beige adipose causes metabolic dysfunction and a subcutaneous to visceral fat switch. Cell 2014, 156, 304-316. [CrossRef] [PubMed]

40. Chen, Y.; Pan, R.; Pfeifer, A. Regulation of brown and beige fat by microRNAs. Pharmacol. Ther. 2017, 170, 1-7. [CrossRef] [PubMed]

41. Zhang, Y.; Li, R.; Meng, Y.; Li, S.; Donelan, W.; Zhao, Y.; Qi, L.; Zhang, M.; Wang, X.; Cui, T.; et al. Irisin stimulates browning of white adipocytes through mitogen-activated protein kinase p38 MAP kinase and ERK MAP kinase signaling. Diabetes 2014, 63, 514-525. [CrossRef] [PubMed]

42. Wiig, H.; Aukland, K.; Tenstad, O. Isolation of interstitial fluid from rat mammary tumors by a centrifugation method. Am. J. Physiol. Heart Circ. Physiol. 2003, 284, H416-H424. [CrossRef] [PubMed]

43. Reiling, E.; Ling, C.; Uitterlinden, A.G.; Van't, R.E.; Welschen, L.M.; Ladenvall, C.; Almgren, P.; Lyssenko, V.; Nijpels, G.; van Hove, E.C.; et al. The association of mitochondrial content with prevalent and incident type 2 diabetes. J. Clin. Endocrinol. Metab. 2010, 95, 1909-1915. [CrossRef] [PubMed]

44. Fernández-Galilea, M.; Pérez-Matute, P.; Prieto-Hontoria, P.L.; Houssier, M.; Burrell, M.A.; Langin, D.; Martínez, J.A.; Moreno-Aliaga, M.J. $\alpha$-Lipoic acid treatment increases mitochondrial biogenesis and promotes beige adipose features in subcutaneous adipocytes from overweight/obese subjects. Biochim. Biophys. Acta 2015, 1851, 273-281. [CrossRef] [PubMed]

(C) 2017 by the authors. Licensee MDPI, Basel, Switzerland. This article is an open access article distributed under the terms and conditions of the Creative Commons Attribution (CC BY) license (http://creativecommons.org/licenses/by/4.0/). 